\title{
Bandwidth Reservations in Home Networks: Performance Assessment of UPnP-QoS V3
}

\author{
Jelle Nelis, Dieter Verslype, Chris Develder \\ Ghent University - IBBT \\ Dept. of Information Technology - IBCN \\ Ghent, Belgium \\ Email: \{jelle.nelis, dieter.verslype, chris.develder\}@intec.ugent.be
}

\author{
Lukasz Brewka, Henrik Wessing, Lars Dittmann \\ Department of Photonics Engineering \\ Technical University of Denmark \\ Kgs. Lyngby, Denmark \\ Email: $\{1 \mathrm{jbr}$, hewe, ladit $\} @$ fotonik.dtu.dk
}

\begin{abstract}
In order for service providers to provide their users high quality services in the home network, Quality of Service $(\mathrm{QoS})$ provisioning is needed to protect premium services. In this paper, we describe how a Universal Plug-and-Play (UPnP) based home network architecture solves this problem in a heterogeneous home network. We outline how it both relieves the end user from troublesome configuration and still offers control to the service provider. We particularly present performance assessment results for UPnP-QoS v3, based on a fully operational experimental implementation. The quantitative measurement results are further used in extensive simulations demonstrating acceptable response times and clear QoS admission differentiation.
\end{abstract}

\section{INTRODUCTION}

Because of the rapid growth of available bandwidth at the user's premises, a wide range of services became accessible. Furthermore, it allowed an increase of user generated content and services. In a situation where the access link is not a narrow bottleneck anymore, the management of home network resources becomes an important issue, especially in light of high bandwidth multimedia applications. One of the challenges in providing end-to-end Quality of Service (QoS) is that the home network is not entirely controlled by the service provider or network operator. Thus, there is a need for a QoS management framework in the home network.

Recently various home networking technologies emerged that support bandwidth reservation (e.g., HomePlug AV, IEEE 802.11e, HomePNA, MoCA). To be able to control end-toend QoS in a heterogeneous home network comprising one or more segments, a common framework is needed to provide an interface to actual physical properties of the network. This is addressed by the UPnP-QoS framework, as discussed in this paper.

In this paper, we present an architectural solution to solve the service provider's problem of dynamically providing QoS guarantees up to and including the home network. In particular, this comprises UPnP-QoS v3, for which we present performance measurement results on our proof-of-concept implementation [1]. In addition, we assess the viability of UPnPbased QoS differentiation through extensive simulations.

\section{Related work}

In the access networks well-known mechanisms such as (G)MPLS ((Generalized) Multi-Protocol Label Switching) can be used to setup the necessary reservations, e.g. based on Carrier Ethernet [2]. Providing these reservations in both backbone and access has been successfully addressed, even considering variations in the required bandwidth [3], [4].

In the home however, the provider has limited control and the complexity of dealing with a heterogeneous home network comprising multiple networking technologies, arises. While this could be addressed with a layer-2 based approach [5], we can capitalize on numerous attempts aiming at the automation of the home network; DPWS [6], IGRS [7], Bonjour [8], Jini [9]. While Bonjour does not consider QoS explicitly, and Jini together with IGRS focus more on end-devices' resource management, DPWS and UPnP are explicitly describing network QoS mechanisms. Early versions of the UPnP-QoS specifications were described in [10], the authors present a possible solution based on UPnP-QoS v1 and Remote Management in Diffserv (RMD). Non-standardized extensions towards parameterized QoS, providing absolute guarantees rather than (relative) prioritization, are proposed in [11]. In [12] extensions to UPnP-QoS v2 for monitoring are proposed and temporal scaling (frame rate reduction) as video adaption technique is considered. The authors of [13] present a distributed video game streaming system relying on UPnP-QoS to overcome network performance issues and [14] proposes a modification to UPnP A/V aiming at enabling multicast of HD content.

The authors of [15] point out the importance of QoS provisioning in the home network and address them on the MAC layer in 802.11. In [16] the authors propose enhancements for the IMS (IP Multimedia Subsystem) QoS framework using SIP (Session Initiation Protocol) information to issue reservations in the home. The design idea of home appliances control service based on DPWS is proposed in [17], where automatic detection of device QoS parameters is addressed.

This paper advances by showing the performance of (to our knowledge) the first complete UPnP-QoS v3 implementation. Our models allow verification of the differentiation level among the different priority classes and enable easy usability assessment of future functionalities.

The paper is structured as follows: in section II we introduce an end-to-end QoS architecture and particularly focus on the components in the home network. Section III presents our 
UPnP-QoS v3 implementation, including measurements and performance optimizations. These measurement data are used as a basis for a simulation study assessing QoS setup metrics in section IV. We summarize our conclusions in section V.

\section{Architecture}

We present an architecture for a service provider to manage in-home network resources for services they provide. The local network resource reservation mechanism is responsible for inhome network resources, and can be remotely managed by the service provider through the use of a remote management protocol. Reservation of resources in the home network ensures qualitative services. By enforcing policies, the service provider can guarantee premium services like Video on Demand or local multimedia streaming to be protected against services that have less stringent QoS constraints.

One of the major problems when managing in-home network resources, is the heterogeneity of the networking technologies used. Home networks grow naturally, users can buy wireless access points to extend their network to include a laptop, but can as easily opt for powerline communication. As opposed to the access network where one single party controls the resources, the home network can be composed of multiple networking segments. All these segments can have different admission mechanisms, this problem needs to be solved by the local QoS management framework. In this paper, we focus on UPnP-QoS.

Within UPnP-QoS, the QosManager (QM) service is responsible for the end-to-end decomposition of QoS requirements and delegation of the admission request. The QosPolicyHolder $(\mathrm{QPH})$ service serves as a policy database the QM can query, while the QosDevice (QD) service acts as an abstraction layer between UPnP-QoS and a specific admission mechanism.

Fig. 1 shows the actions involved in a UPnP-QoS request. The UPnP-QoS Control Point, i.e. the requesting application, invokes a QM service on the network. Information about the identity of the stream together with its QoS requirements are passed.

First, the QM entity will query all QD services for path information so the path the stream traverses can be calculated.

Once the path is known, the QoS state of all QD services on the path will be retrieved after which the actual admission will take place. The underlying admission mechanisms of each QD service will decide whether or not the stream can be admitted.

In case of failure, a couple of things can happen depending on the parameters the application provided. The application can indicate to do preemption or to only report which streams on the network are currently blocking reservation of resources. Fig. 1 shows what happens in case of preemption. First of all, the policies for all blocking streams (which were returned as a result of the failed QD:AdmitTrafficQos() action) are retrieved from the QPH service. Based on the UserImportanceNumber (UIN) of each of the streams, QoS for the preemption candidates is released after which admission for the current stream is tried again.

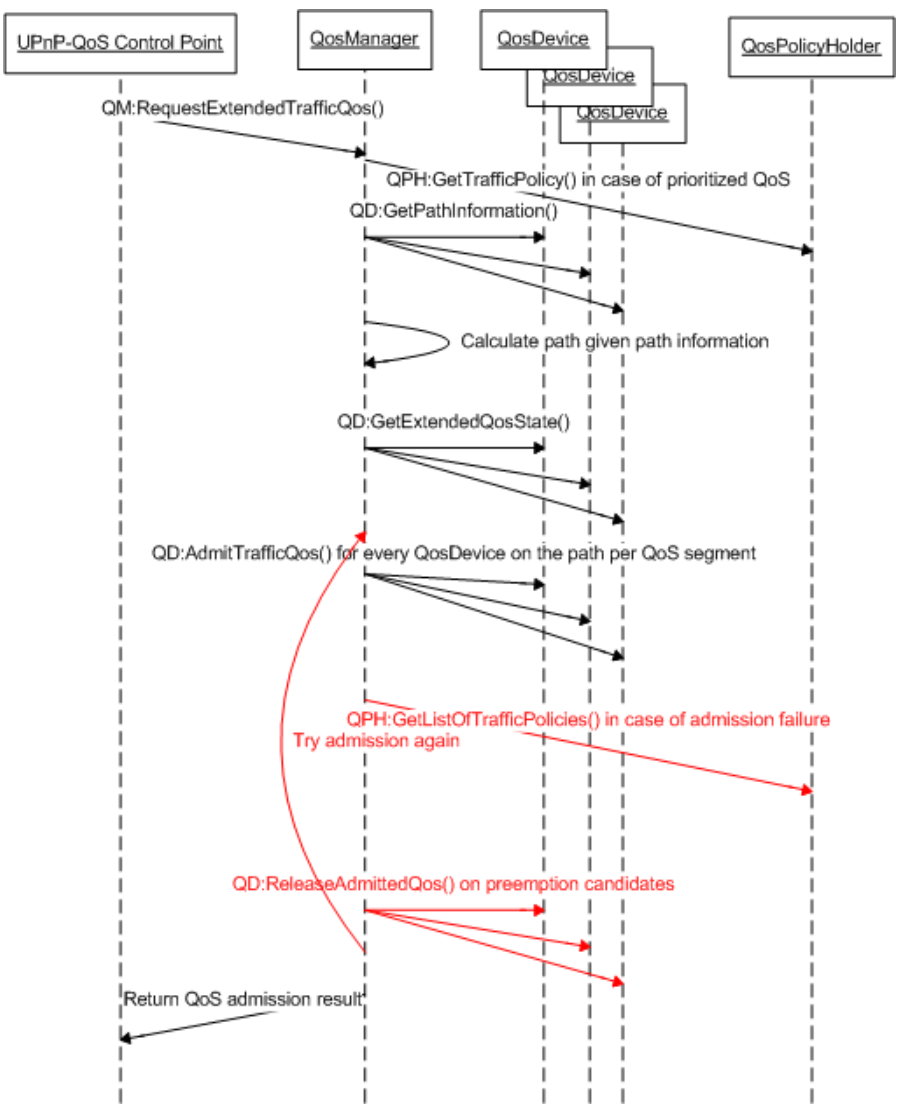

Fig. 1. Sequence diagram of QoS request with optional contention resolution (preemption)

\section{EXPERIMENTAL IMPLEMENTATION}

Ghent University - IBBT has implemented the complete UPnP-QoS v3 specifications together with a framework that simplifies the implementation of QosDevice services (for details see [18]). To highlight the necessary steps for reliable, policy based resource reservation on a heterogeneous home network, we successfully demonstrated the following scenarios in a UPnP-QoS v3 over MoCA [19] demonstration [1].

1) Basic QoS reservation: This scenario shows that the differences in technological capabilities can be abstracted to offer a unified resource reservation mechanism across the home network (see Fig. 2). QoS is requested for a stream originating outside of the home network. Due to the fact QoS has been reserved for the VoD stream, the best effort IP traffic within the home network will be restricted to the remaining bandwidth. This scenario covers all communication up to admission on the QD services.

2) Resource contention: In a seconds scenario (depicted in Fig. 3) resource contention is solved based on predefined policies, and resources are released to make room for the reservation at hand.

Each UPnP-QoS action has been tested, each run consisted of one hundred consecutive invocations. Table I shows the average times of the performed tests. Note that parsing refers to processing of the action responses received by 


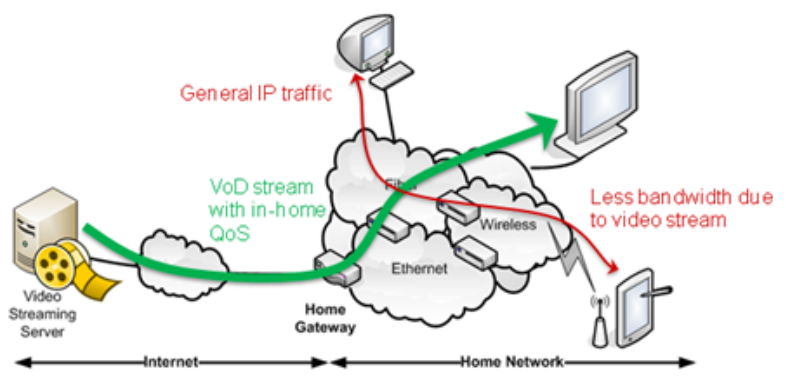

Fig. 2. Basic QoS reservation use case

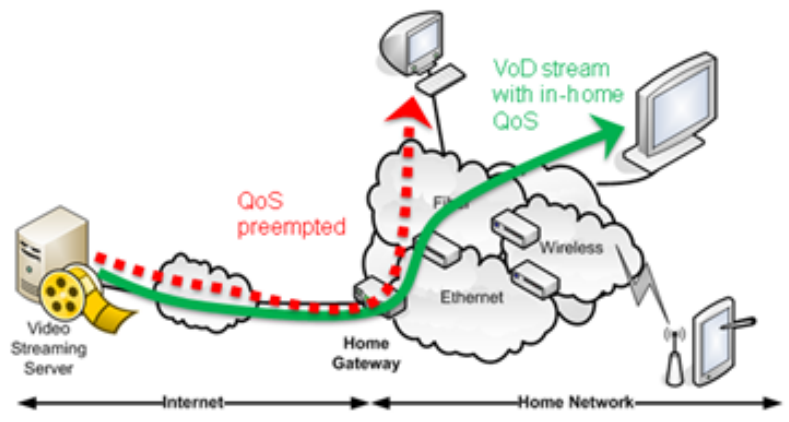

Fig. 3. Resource contention use case

TABLE I

INVOCATION TIMES, I.E. RESPONSE TIMES AND PARSING, FOR UPNP QOSDEVICE ACTIONS ON MOCA IMPLEMENTATION: (I) GPI GeTPATHINFormation, (II) GEQS: GetExTENDEDQOSSTATE, (III) ATQ: AdmitTrafFicQos, AND (IV) RAQ: RelEAsEAdmitTedQos.

\begin{tabular}{l||c|c|c|c} 
MoCA node & GPI & GEQS & ATQ & RAQ \\
\hline Network Coordinator & $25 \mathrm{~ms}$ & $110 \mathrm{~ms}$ & $429 \mathrm{~ms}$ & $72 \mathrm{~ms}$ \\
parsing: & $7 \mathrm{~ms}$ & $18 \mathrm{~ms}$ & - & - \\
\hline non-Network Coordinator & $18 \mathrm{~ms}$ & $110 \mathrm{~ms}$ & $908 \mathrm{~ms}$ & $120 \mathrm{~ms}$ \\
parsing: & $7 \mathrm{~ms}$ & $19 \mathrm{~ms}$ & - & -
\end{tabular}

the QosManager entity. Clearly, parsing times are only nonneglible for the GPI and GEQS actions, since their responses contain a significant amount of state information from the QosDevice.

It is interesting to note that MoCA uses a Network Coordinator (NC) to perform the actual admission. Our measurements indicate that the response times significantly depend on whether or not the reservation request is initiated by the NC in the MoCA 1.1 network. As expected no real difference is observed between the results for GPI and GEQS on a NC and a non-NC since no interaction with the MoCA network is needed. The results for ATQ and RAQ however, reveal an interesting observation: there is a considerable penalty for having to contact the NC. This observation is valid both for requesting as for releasing resources.

\section{Simulation anAlysis}

\section{A. Model}

The topology of the model developed for the purpose of the simulations is presented in Fig. 4. We assume the QM, QPH and CP functionality are implemented on a single node, issuing the QoS requests. This node (e.g. a home gateway) is interconnected with three end QDs by an intermediate QD with switching functionality. All the links in the model provide $70 \mathrm{Mbps}$ full duplex connectivity. The $\mathrm{CP}$ generates requests in exponentially distributed intervals with a mean value used as a parameter of the simulations. The requests are uniformly distributed between four priority groups. The data plane resources being reserved are randomly assigned a TrafficImportanceNumber (0-7) and are uniformly distributed in a range of values between 2.5 and $10 \%$ of the link bandwidth. The source and destination of the flow are randomly chosen. The holding time of the soft-state reservations is 480 seconds, the simulation time is 200 minutes with a 25 minutes warm-up period. The QD response times used in the model are based on the values for the NC in the MoCA network. The described model was developed using the OPNET modeling tool [20].

\section{B. Simulations}

The motivation behind the performed simulations is to verify the QoS differentiation for requests with different priorities in a dynamic scenario. Fig. 5 represents the QoS request rejection ratio, measured as the number of rejected requests over the total requests, in one of the four classes. It is clearly visible that a good level of differentiation on the signaling level can be achieved using UPnP-QoS. One can notice over $40 \%$ reduction in the rejection for high priority classes compared to low priority classes. This is archived for uniform distribution of traffic in all classes and if required could be easily improved by a more selective classification of traffic to a high priority class.

Lower request rejection values for high priority requests are the consequence of preemption. However, the preemption procedure will obviously prolong the QoS establishment. Fig. 6 presents the average QoS setup time for different priorities. The increase of setup time due to preemption is reflected in different setup times across the request priorities. As higher priority streams have more chance of triggering the preemption procedure, higher priority stream will have a higher average



Fig. 4. Topology of modeled UPnP QoS enabled network 


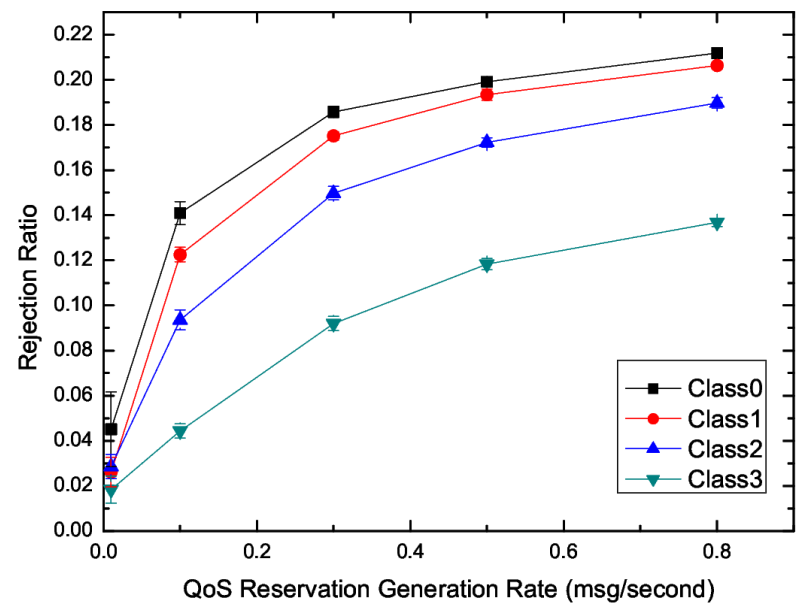

Fig. 5. Rejection ratio for different priority flows as a function of the flow initiation rate

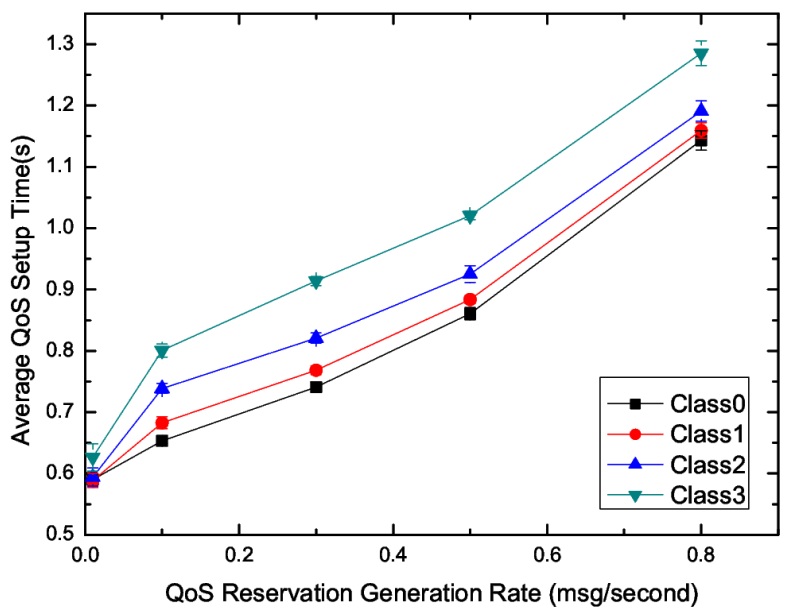

Fig. 6. Setup time for flows of different priority in function of traffic QoS request message generation rate

setup time. The results also show that an increase of the CP's request rate causes longer setup times. This is caused by the growing probability of preemption because of higher resource utilization.

All the graphs present the results with $90 \%$ confidence intervals.

\section{CONCLUSION}

To solve the problem of offering QoS control even within the (heterogeneous) home network, we propose to use a plugand-play approach using UPnP-QoS. In this paper, we have presented quantitative performance assessment results based on the first complete implementation of the recent UPnP-QoS v3 standard on MoCA devices. Extensive simulation results, using that measurement data, proved clear QoS differentiation and acceptable response times (in the order of one second for a home network with five QosDevice services involved).

\section{ACKNOWLEDGMENT}

The research leading to these results has received funding from the European Communitys Seventh Framework Programme (FP7) under project 212352 ALPHA "Architectures for fLexible Photonic Home and Access networks". C. Develder is Post-Doctoral Fellow of the Research Foundation Flanders (FWO-Vlaanderen).

\section{REFERENCES}

[1] [Online]. Available: http://ir.entropic.com/releasedetail.cfm?ReleaseID= 402263

[2] L. Fang, R. Zhang, and M. Taylor, "The evolution of carrier ethernet services - requirements and deployment case studies," IEEE Commun. Mag., vol. 46, no. 3, pp. 69-76, Mar. 2008.

[3] T. Anjali, et al., "Dynamic bandwidth reservation for label switched paths: An on-line predictive approach," Computer Communications, vol. 29, no. 16, pp. 3265 3276, 2006, more Than a Protocol for Next Generation Internet. [Online]. Available: http://www.sciencedirect.com/science/ article/B6TYP-4K4PVVY-1/2/df9038e6aeca83161a74bfebd2ace2f5

[4] M. E. Hachimi, et al., "Dynamic bandwidth allocation in sip based mpls," in AINA '07: Proceedings of the 21st International Conference on Advanced Networking and Applications. Washington, DC, USA: IEEE Computer Society, 2007, pp. 917-923.

[5] J.-P. Javaudin et al., "Inter-MAC concept for gigabit home networks," in Proc. Workshop on Inter-Mac Management and Multiple interfaces for Multi-Gigabit Wireless Systems (MGWS), part of IEEE Int. Symp. on Personal, Indoor and Mobile Radio Communications (PIMRC 2009), Tokyo, Japan, 13-16 Sep. 2009.

[6] Devices Profile for Web Services Version 1.1, OASIS, http://docs.oasisopen.org/ws-dd/dpws/wsdd-dpws-1.1-spec.html, July 2009.

[7] Internet Grouping and Resource Sharing, IGRS Information Industry Association, http://www.igrs.org/en/index/index.asp.

[8] Bonjour Overview, Apple Inc., http://developer.apple.com/ documentation /Cocoa/Conceptual/NetServices/NetServices.pdf, May 2006.

[9] Jini Architecture Specification, http://www.jini.org/wiki/Jini_ Architecture_Specification, March 2007.

[10] J.-L. Chen, M.-C. Chen, and Y.-R. Chian, "QoS management in heterogeneous home networks," Computer Networks, vol. 51, no. 12, pp. 3368 - 3379, 2007. [Online]. Available: http://www.sciencedirect.com/science/article/ B6VRG-4N0X5YT-3/2/40a28abd715af797a611f45663de625b

[11] R. Seepold, N. M. Madrid, and J. M. Fernández, "QoS management for distributed multimedia services," in Proc. 10th IFIP/IEEE Int. Conf. on Management of Multimedia and Mobile Networks and Services (MMNS 2007), San Jose, CA, Oct. 31-Nov. 22007.

[12] H. Lee, S. Moon, and J. W. Kim, "Enhanced UPnP QoS architecture for network-adaptive streaming service in home networks," IEEE Trans. on Consumer Electronics, vol. 53, no. 3, pp. 898-904, Aug. 2007.

[13] T. Jurgelionis, et al., "Distributed video game streaming system for pervasive gaming," in Proc. STreaming Day 2009, Genova, Italy, 21 Sep. 2009.

[14] T. Hwang, H. Park, and J. W. Chung, "Home-to-home media streaming system based on adaptive fastreplica," in Advanced Communication Technology, 2009. ICACT 2009. 11th International Conference on, vol. 03, feb. 2009, pp. $1665-1666$.

[15] Y. He, et al., "A reservation based backoff method for video streaming in 802.11 home networks," Selected Areas in Communications, IEEE Journal on, vol. 28, no. 3, pp. $332-343$, april 2010.

[16] R. Good and N. Ventura, "End to end session based bearer control for ip multimedia subsystems," in Integrated Network Management, 2009. IM '09. IFIP/IEEE International Symposium on, june 2009, pp. $497-504$.

[17] C.-M. Huang, H.-H. Ku, and C.-W. Lin, "Design and implementation of a web2.0-based home-appliances control service platform," in Pervasive Systems, Algorithms, and Networks (ISPAN), 2009 10th International Symposium on, dec. 2009, pp. $110-115$.

[18] C. Develder et al., "Delivering scalable video with QoS to the home," Telecommun. Syst., 2010, in press.

[19] [Online]. Available: http://www.mocalliance.org/

[20] OPNET Modeler Version 14.5.A, http://www.opnet.com, March 2007. 